\title{
Two-photon Imaging of Synaptic Plasticity and Pathology in the Living Mouse Brain
}

\author{
Jaime Grutzendler* and Wen-Biao Gan ${ }^{\dagger}$ \\ *Northwestern University \\ 303 East Chicago Avenue, Ward Building 10-132 \\ Chicago, IL 60611 and \\ ${ }^{\dagger}$ Skirball Institute of Biomolecular Medicine, \\ Department of Physiology and Neuroscience \\ New York University School of Medicine, \\ 540 First Avenue, New York, NY 10016
}

\begin{abstract}
Summary: Two-photon microscopy (TPM) has become an increasingly important tool for imaging the structure and function of brain cells in living animals. TPM imaging studies of neuronal structures over intervals ranging from seconds to years have begun to provide important insights into the structural plasticity of synapses and the modulating effects of experience in the intact brain. TPM has also started to reveal how neuronal connections are altered in animal models of neurodegeneration, acute brain injury, and cerebrovascular disease.
\end{abstract}

Here, we review some of these studies with special emphasis on the degree of structural dynamism of postsynaptic dendritic spines in the adult mouse brain as well as synaptic pathology in mouse models of Alzheimer's disease and cerebral ischemia. We also discuss technical considerations that are critical for the acquisition and interpretation of data from TPM in vivo. Key Words: Two-photon microscopy, imaging, dendritic spines, synapses, Alzheimer's disease, cerebrovascular disease.

\section{INTRODUCTION}

Changes in synaptic connections occur throughout the life of an animal. During development, synaptic connections are rapidly formed and eliminated to establish a functional brain that in part reflects the experience of early life. ${ }^{1-5}$ In adulthood, the nervous system retains some capacity to reorganize its connections following peripheral and central alterations of inputs. ${ }^{6-12}$ Such rearrangements of neuronal connections are likely to be essential for the rehabilitation of the central nervous system (CNS) following acute and chronic injury. ${ }^{13}$

Until recently, much of our knowledge about synaptic structural alterations in the intact brain had been inferred from single-time-point observations using fixed preparations. Due to the complexity and variability of the CNS,

\footnotetext{
${ }^{\dagger}$ Address Correspondence and reprint requests to: Wen-Biao Gan, Ph.D., Skirball Institute of Biomolecular Medicine, Department of Physiology and Neuroscience, New York University School of Medicine, 540 First Avenue, New York, NY 10016. E-mail: gan@ saturn.med.nyu.edu.
}

it is often difficult, if not impossible, to extrapolate from studies in fixed preparation the degree of structural change of synapses, especially when little or no net change in the number or size of synapses occurs. It is evident that the best way to examine structural plasticity of synapses is by following individual synapses over time in living animals. Such an approach has revealed fundamental insights into the plasticity of synapses in the peripheral nervous system and their sculpting over development. ${ }^{14-16}$ Technical advances have recently made it feasible to image individual synapses over extended periods of time in the CNS. In particular, the advent of Green Flourescent Protein (GFP) and its spectral variants and the ability to generate transgenic mice or viral vectors to drive the expression of such proteins in specific cell types in the nervous system ${ }^{17-19}$ are allowing for long-term imaging of individual synaptic structures at high resolution in living animals. ${ }^{18,20-23}$

The other major technical advance relates to better instrumentation for optical imaging of living tissues. Specifically, two-photon microscopy (TPM) has dra- 

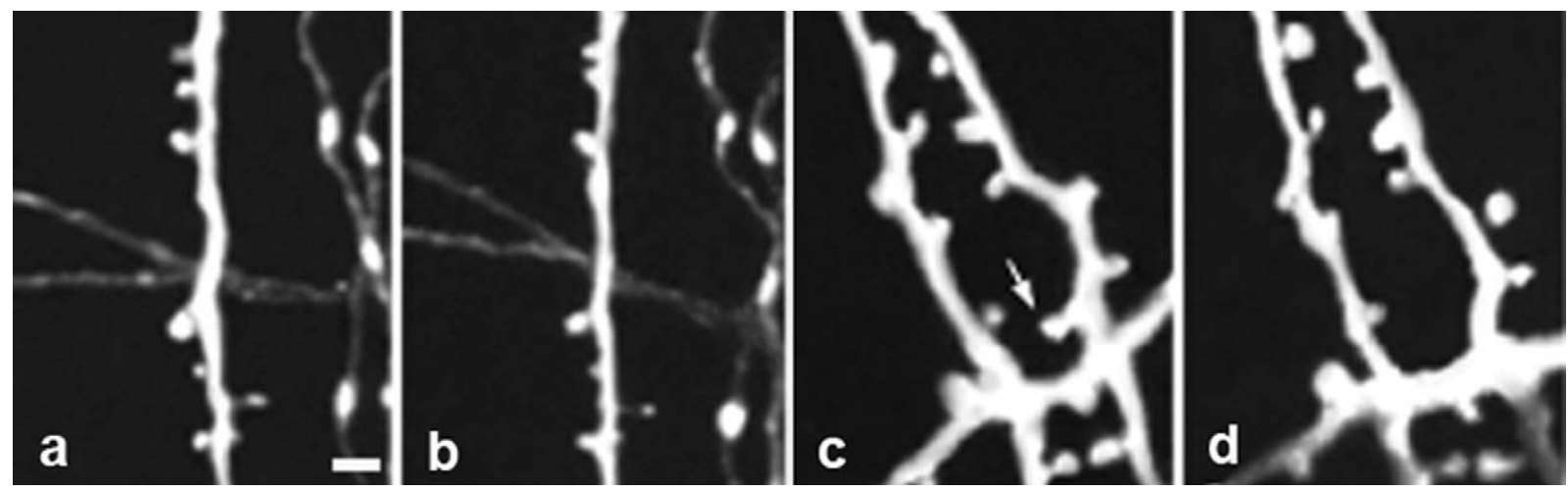

FIG. 1. Dendritic spines in adult mice demonstrate long-term stability. a-b Dendritic branches imaged three days apart show the same spines at the same locations. c-d. Repeated imaging of dendritic branches imaged 2 months apart show little change in spine number or location. The arrow in (c) points to a spine that is eliminated in the second view (d). Scale bar $1 \mu \mathrm{m}$ for a-d. Modified from Grutzendler et al. ${ }^{21}$ with permission.

matically enhanced the ability of deep tissue imaging. ${ }^{24}$ Unlike one-photon excitation in confocal microscopy, two-photon excitation depends on nearly simultaneous absorption of two photons by a single fluorophore. For thick specimens and vital imaging, TPM has several advantages over confocal laser scanning microscopy. Two-photon microscopy significantly reduces light absorption and scattering within biological specimens due to the use of far- or infra-red photons for the excitation of fluorophores. In addition, the absence of excitation out of the plane of focus in TPM minimizes both photobleaching and photodamage. This is very important for monitoring living cells because prolonged light exposures often cause significant photo-toxicity. Moreover, because a confocal pinhole is no longer needed and the emission fluorescence is not required to pass back through the scanning mechanism as in the confocal microscope, the detection of emitted light can be more efficient in TPM. Thus, TPM allows imaging of thick biological specimens such as brain slices, intact embryos, and brains up to $\sim 800 \mu \mathrm{m}$ deep into the tissues. ${ }^{25,26}$

Rather than reviewing the extensive field of structural synaptic plasticity on which there are many excellent imaging studies and reviews, ${ }^{3,18,23,27-29}$ in this paper we will mainly focus on recent findings obtained by TPM in living mice that relate to structural changes of synapses in the normal and degenerating brain. Because live imaging of synapses is a relatively new field, there is still no consensus regarding the degree of synaptic dynamism at baseline and in response to experience. Many of these discrepancies between studies are likely to be explained by methodological differences. We, therefore, also discuss technical issues and considerations that are critical for the acquisition and interpretation of TPM data in the cortex of living mice.

\section{IMAGING SYNAPTIC STRUCTURAL PLASTICITY IN THE YOUNG AND ADULT MOUSE BRAIN}

\section{Plasticity of postsynaptic dendritic spines under laboratory housing conditions}

Dendritic spines are the postsynaptic sites of excitatory axo-dendritic synapses in the CNS and their structural changes therefore serve as a good indicator of synaptic plasticity. ${ }^{30}$ In vivo TPM imaging studies so far have mainly focused on examining the degree of dendritic spine elimination and formation. In the adult mouse primary visual cortex ( $>4$ months of age), repeated imaging over days to months showed that spines in apical dendrites from layer $\mathrm{V}$ pyramidal neurons are remarkably stable with $\sim 4 \%$ turnover per month and a half-life $>13$ months (FIG. 1). ${ }^{21}$ In addition, this study also showed that in mice between 1 to 3 months of age, the rate of spine elimination is significantly higher than that of spine formation, leading to a net loss of spines during young adolescence. These results suggest that a significant percentage of spines in the mature adult mouse primary visual cortex can last throughout the lifespan of an animal and thus could provide a structural basis for lifelong information storage. ${ }^{21}$ A different study of TPM imaging in adult mouse barrel cortex, however, suggested that spines are highly plastic in the adult brain: $\sim 20 \%$ of spines turn over within one day and $\sim 40 \%$ of spines turn over within a week. ${ }^{22}$ Thus, the first two studies using in vivo imaging of GFP expressing dendritic spines generated fundamentally different views on the stability of synapses in the adult nervous system. ${ }^{31,32}$ Follow-up studies continue to show substantial differences in spine dynamics. Holtmaat et al. ${ }^{33}$ found that the high spine turnover rate previously reported in barrel cortex ${ }^{22}$ is partly due to the use of young adult mice (6 to 10 weeks of age) instead of mature adults. Although this 
newer study showed the majority of adult spines can be stable over weeks, more than $20 \%$ of total spines in 6-months-old mice are still considered transient and persist less than 4 days. ${ }^{33}$ On the other hand, Zuo et al. ${ }^{34}$ showed that in adults ( $>4$ months old), spines exhibited similar low rates of elimination and formation in barrel, primary motor, and frontal cortices as their previously reported low turnover rates in the primary visual cortex. They also showed that in adult barrel cortex, $>70 \%$ of spines are persistent over an 18-month interval, underscoring the remarkable stability of dendritic spines in adulthood and their potential role in long-term information storage. ${ }^{34}$ A recent study examining spine dynamics in the visual, somatosensory, and auditory cortices in $\sim 2$ month-old mice found that $>80 \%$ of spines are stable over 3 weeks, ${ }^{35}$ in agreement with the view that adult spines are largely stable.

It is important to note that the above studies mainly imaged spines in apical dendrites of layer $\mathrm{V}$ pyramidal neurons. Studies in other brain areas such as the hippocampus and the olfactory bulb have demonstrated high levels of stability of adult dendritic branches and spines over hours to days. ${ }^{36,37}$ Recently, a study of aspiny interneurons in adult mouse visual cortex showed that while few branches demonstrated a relatively high degree of dynamism, the majority of dendritic branches were stable over weeks. ${ }^{38}$ Thus, despite the differences in the various studies, it can be concluded that under laboratory housing conditions, a large percentage of dendritic branches and spines are highly stable in adulthood and likely able to persist for the duration of a mouse's life.

\section{Experience-dependent plasticity of dendritic spines}

It is well established that experience profoundly influences synaptic connectivity and behavior throughout life. ${ }^{1-3,6,39}$ In the developing nervous system, for example, experience-dependent competition among axonal inputs plays an important role in eliminating some connections while expanding others. ${ }^{2,3,6}$ Recently, TPM has been used to examine the long-term effect of sensory experience on the rates of dendritic spine elimination and formation in mouse barrel cortex, a cortical region in which sensory input can be easily manipulated. By trimming all the whiskers on one side of the mouse facial pad daily, Zuo et al. ${ }^{40}$ showed that during the second postnatal month when extensive spine loss occurs, sensory deprivation preferentially reduces the rate of spine elimination but not formation. In contrast, no significant difference in spine elimination was found in adult mice over two weeks, suggesting that the impact of sensory deprivation on spine elimination diminishes as animals reach adulthood.

The effect of sensory experience on spine elimination and formation has also been examined by trimming ev- ery other whisker in a chessboard pattern on one side of the facial pad. Unlike trimming all the whiskers which deprives all sensory inputs, chessboard deprivation removes only half of the sensory inputs and increases the difference in sensory experience coming from adjacent whiskers. Trachtenberg et al. ${ }^{22}$ tested the effect of 4-day chessboard deprivation on spine dynamics and found that overall spine density was not affected but spine turnover increased significantly in 5 to 10 week-old mice. This suggests that chessboard deprivation leads to rapid increase in both elimination and formation of dendritic spines in young adult mice. In another study, Zuo et al. ${ }^{40}$ found that similar to all-whisker trimming, chessboard trimming over two weeks preferentially reduces spine elimination without effect on spine formation in young adolescent mice. The effect of chessboard trimming, however, is less robust with all-whisker trimming. Importantly, chessboard trimming over 2 weeks had no significant impact on spine dynamics in adult mice. Together, these studies suggest that sensory deprivation has profound effects in synapse structural plasticity in young somatosensory cortex but plays a limited role in modifying spine turnover in adulthood. Consistent with this view, manipulation of neuronal activity in olfactory bulb and sensory inputs to auditory cortex have little or no effects on the stability of adult dendritic branches and spines over hours to days. ${ }^{35,37}$ The limited role of experience in modifying the turnover of stably connected adult synapses may have important implications in the slow rehabilitation of the adult CNS following injury.

\section{IMAGING STRUCTURAL PLASTICITY OF SYNAPSES IN MOUSE MODELS OF ALZHEIMER'S AND CEREBROVASCULAR DISEASES}

\section{TPM imaging of synaptic pathology in mouse models of Alzheimer's disease}

Functional and structural changes of synapses are thought to occur early in the pathogenesis of several neurodegenerative diseases. This is especially true in Alzheimer's disease where the best correlate of cognitive dysfunction is thought to be the loss of synapses as seen by immunohistochemistry and electron microscopy in post mortem tissue. Fibrillar amyloid $(\mathrm{A} \beta)$ deposition, one of the hallmarks of $\mathrm{AD}$, is commonly associated with dystrophic neurites ${ }^{41-43}$ and aberrant sprouting, ${ }^{44-47}$ as well as increased curvature of dendritic processes. ${ }^{48-50}$ However, only $\mathrm{A} \beta$ deposition in certain areas but not others correlates well with the degree of cognitive dysfunction. ${ }^{46,51-55}$ Furthermore, in transgenic mouse models of $\mathrm{AD}$ it has been shown that cognitive dysfunction may occur even before plaque deposition. ${ }^{56}$ This and other findings have raised the possibility that soluble 
forms of amyloid oligomers can have an important role in initiating the disease possibly by targeting synapses. ${ }^{57}$

With the advent of TPM, it is now possible to monitor directly in living mice the disruption of neuronal circuits in the progression of $\mathrm{AD}$ neuropathology. By crossbreeding a transgenic mouse model of $\mathrm{AD}$ with mice expressing GFP in subsets of neurons, Tsai et al. ${ }^{58}$ studied the degree and time course of synaptic disruption associated with fibrillar amyloid deposits in vivo with TPM. They monitored dendrites passing through, or in the vicinity of amyloid deposits and found that these dendrites exhibit spine loss and shaft atrophy whereas nearby axons develop large varicosities. Time-lapse imaging over days to weeks revealed extensive formation and elimination of dendritic spines and axonal varicosities within a radius of $\sim 15 \mu \mathrm{m}$ from the periphery of the fibrillar deposit, suggesting that amyloid deposition triggers continuous remodeling of nearby neuronal structures (FIG. 2). Furthermore, local neuritic abnormalities associated with amyloid deposits result in eventual breakage of dendrites and axons, leading to large-scale and permanent disruption of neuronal connections. A similar study ${ }^{59}$ using in vivo imaging of neurons labeled by viral vector expression of GFP in a different transgenic AD mouse model also found extensive synaptic abnormalities associated with dense core amyloid plaques. These studies indicate that the accumulation of fibrillar amyloid is far more detrimental to neuronal circuitry than previously thought, and underscore the importance of therapies aimed at early plaque clearance or preventing amyloid deposition.

As active and passive immunization involving antibodies against $\mathrm{A} \beta$ peptides have been proposed as a potential therapeutic modality for $\mathrm{AD}$, many studies have investigated the effects of immunotherapy on amyloid plaques and the effects of plaque removal on the surrounding neuronal structures using fixed preparations. A recent study has used TPM to follow the dynamics of axonal and dendritic varicosities around amyloid plaques before and after immunotherapy. ${ }^{43}$ They found that exposure of the mouse cortex to anti-A $\beta$ antibodies lead to the disappearance of some of the varicosities. It remains to be explored whether this result implies that the therapy was beneficial in that it presumably lead to a reversal in the process of varicosity formation or instead varicosities disappeared more rapidly because the treatment accelerated the process of neuritic disruption.

It is worth noting that amyloid plaques are typically surrounded by activated microglia and astrocytes. The role of these cells in the disease process remains controversial. Some studies have suggested that microglia have protective roles while others suggest that microlgia may have a role in causing secondary neuronal injury. ${ }^{60}$ Furthermore, it is not known what role they play in the process of amyloid deposition. With the availability of mice that express fluorescence in microglia ${ }^{61}$ or astro-
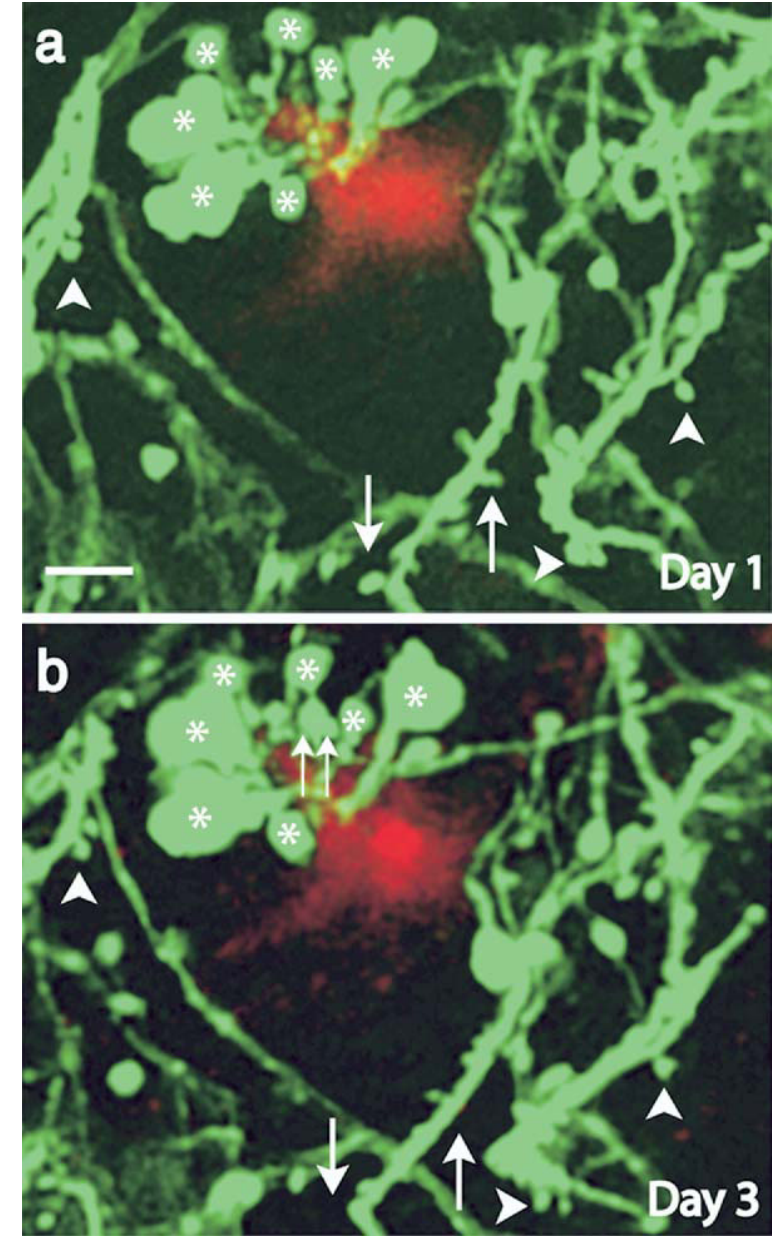

FIG. 2. Axonal and dendritic abnormalities near fibrillar deposits occur progressively over days to weeks. (a, b). In vivo time-lapse imaging of cortical dendrites and axons (green) near an $A \beta$ deposit (red) in cortical layer 1 of PSAPP/YFP mice at 6 months of age. While the majority of spines (arrowheads) and varicosities (asterisks) were stable over 2 days, some structural changes (e.g., spine loss (arrows) and varicosity formation (double arrows)) did occur. Scale bar $=5 \mu \mathrm{m}$. Data from Tsai et al. ${ }^{58}$ with permission.

cytes, ${ }^{62}$ it should be possible to simultaneously image with TPM amyloid plaques, microglia, and neuronal structures. ${ }^{63}$ These types of studies are likely to provide a wealth of information regarding the spatio-temporal relation between plaque formation, microglia migration around plaques, and the resulting synaptic pathology.

\section{In vivo imaging studies of neuronal circuit disruption in models of cerebral ischemia}

Cerebral ischemia is a prevalent clinical entity with devastating consequences. However, despite the poor long-term outcomes following stroke, a significant proportion of patients experience spontaneous neurological improvement. The cellular mechanisms that underlie the neurological dysfunction and recovery following ischemia are poorly understood. Resolution of neurological deficits after stroke might occur 
through changes in the structure and function of surviving neurons including synaptic reorganization, axonal sprouting, and neurogenesis. The study of such complex cellular processes has been limited by our inability to repeatedly imaging neuronal structures at high resolution in living animals receiving stroke models. Two-photon microscopy has now partially resolved these obstacles by allowing the acquisition of time lapse images of the same cellular structures over intervals of up to months in living mice.

Focal stroke is known to produce a central area of cell death, surrounded by areas with different degrees of neuronal injury due to variable reduction in blood flow as a result of collateral circulation (Penumbra). It is thought that in the "Penumbra" cells may undergo delayed cell death and significant changes in neuronal connectivity that may be partially responsible for the functional recovery seen after cerebral ischemia. Several studies have recently found changes in neuronal structures in particular dendritic spines following different models of cerebral ischemia. ${ }^{64,65}$ Zhang et al. ${ }^{64}$ used several models of stroke in which different degrees of blood flow reduction were observed. A severe blood flow reduction with a photothrombosis stroke model leads to a very rapid spine and dendritic disruption. In contrast, with moderate blood flow reduction by administration of the vasoconstrictor endothelin no changes in dendritic spine structure were observed over 5 hours suggesting that minimal neuronal circuit disruption took place. This could potentially explain why therapeutic reperfusion using thrombolytic agents results in significant functional recovery if given early after stroke.

Vascular occlusion by photothrombosis has also recently been used to study flow changes in microcirculation following single vessel obstruction opening the door to studying cerebral hemodynamics in the living mouse cortex. ${ }^{66}$ In addition to studying how neurons and their connections change in response to restricted blood flow, it is now also possible to examine other cell types that are likely to be involved in cerebrovascular regulation such as astrocytes and neuroinflammation such as microglia. Furthermore, crossbreeding mice that express different fluorescent proteins in specific cell types (neurons, astrocytes, and microglia) would allow imaging of cell-cell interactions in vivo. This is likely to be very important for studying the role of microglia in secondary neuronal damage following CNS injury. Finally, neurogenesis and migration of newly born neurons into an area of injury could also constitute a significant mechanism of neural repair following cerebral ischemia. ${ }^{67,68}$ In vivo imaging could provide a useful tool to better understand how these migrating neuroblasts mature and integrate into functional neuronal circuits.
Technical considerations and data interpretation for in vivo imaging of synapses

Although most recent results on the degree of postsynaptic dendritic spine plasticity converge towards the view that the vast majority of spines are stable over months to years, significant discrepancies still exist. In the future, it will be important to resolve these differences because they lead to different interpretations on how information is processed and stored in the brain. Accurate measurement of spine turnover rates is also important for studying synaptic pathology in CNS diseases. Below we discuss several factors such as different imaging approaches and data interpretation that are likely to affect measurements of dendritic spine stability in living animals:

Thin-skull versus cranial window approaches. Methodological differences in the skull preparation could have a major impact on the structural stability of synapses. In general, studies that used a minimally invasive method consisting of imaging through a thinned skull preparation tended to show very low degree of dendritic spine plasticity. ${ }^{21,34}$ In contrast, studies imaging through a cranial window, which involves removal of a piece of skull $\sim 5 \mathrm{~mm}$ in diameter, and covering the exposed brain with agarose and a glass coverslip ${ }^{22,33}$ showed much greater degrees of dendritic dynamism. In the latter case, imaging usually starts 7 to 10 days after placing the cranial window. Presumably, this waiting period plus the administration of systemic corticosteroids are required for the resolution of an initial inflammatory reaction, which impedes imaging within the first weeks after craniotomy. ${ }^{22,33}$ This neuroinflammatory reaction and the unavoidable meningeal vascular injury associated with skull removal are likely to induce significant cortical injury and enhance the degree of spine plasticity.

The limitations of the thin-skull technique are mainly related to the fact that skull thickness is critical for image quality and it takes a lot of practice to consistently obtain a preparation of the optimal thickness for spine imaging. In the authors' experience, to obtain high-resolution images of dendritic spines, the skull must be less than 30 $\mu \mathrm{m}$ thick. It is worth noting that over-thinning the skull can also lead to cortical injury, presumably due to deformation of the skull under the pressure of the blade. In such cases, the thinning process generally leads to mild neuronal injury, mainly manifested by axonal and dendritic blebbing, and eventual disappearance of fluorescent structures. ${ }^{21,34}$ For easy detection of neuronal injury during the thinning process or cranial window implantation, it is therefore helpful to use transgenic mice in which neurons are densely labeled (YFP-H line ${ }^{17}$ ) rather than mice with low neuronal labeling density (GFP-M line $\left.{ }^{17}\right)$.

Animal age. Imaging through thinned-skull showed that in the first few postnatal months, the rate of spine 
elimination is significantly higher than that of spine formation, resulting in a concurrent net loss of spines in layer 5 pyramidal neurons of various cortical regions. These studies showed that adult spine stability is achieved after four months of age. ${ }^{21,34}$ These observations are consistent with previous studies from fixed tissue showing that synaptic density in the mammalian cortex decreases substantially from infancy until puberty and becomes stabilized in adulthood. ${ }^{5,69,70}$ In agreement with this age-dependent spine stabilization, Holtmaat et al. ${ }^{33}$ found that spine turnover in the barrel cortex of mice at six months of age is significantly lower than that in mice at 6 to 10 weeks of age. ${ }^{22}$ These studies underscore the importance of considering the precise age of the animal when studying structural synaptic changes.

Classification of dendritic protrusions. The third factor that partially contributes to differences in measurements of spine stability relates to the distinction between filopodia-like structures and dendritic spines. Many lines of evidence indicate that filopodia and spines, although related, are structurally and functionally different entities. Filopodia are thin and long protrusions without a bulbous head. Unlike spines, filopodia contain little or no AMPA receptors that are important for synaptic transmission. ${ }^{71}$ The lifetime of filopodia in both young and adult cortex is on the order of hours whereas spines have a lifetime of many months. In vitro studies have suggested that filopodia are highly dynamic and are precursors of spines. ${ }^{72-74}$ Consistent with this view, the vast majority of filopodia in vivo were found to undergo rapid turnover within hours and only a small percentage of them are converted to spines, which then last for more than 1 to 2 days. ${ }^{21,34}$ Because filopodia are fundamentally different from spines in structure and function, it is important to measure and quantify their dynamics separately with appropriate temporal resolutions, particularly in young animals in which filopodia are highly abundant. For example, because $\sim 15 \%$ of dendritic protrusions are filopodia and more than $50 \%$ of filopodia turn over within 4 hours in young adolescent barrel cortex, ${ }^{34}$ the total number of filopodia eliminated and formed within 3 days is comparable to the total number of spines. Including filopodia in the same category as spines in the quantification of spine dynamics would suggest that at least $50 \%$ of spines turn over within 3 days. Such a high turnover rate would be an overestimation of the dynamism of the majority of spines.

In summary, it is possible that the discrepancy over spine dynamics in the previous studies results from a combination of factors including different imaging techniques and animal ages as well as mixing filopodia and spines in data quantification. Future studies are required to further address the contribution of these various factors to spine dynamics. As pointed out by others, ${ }^{75}$ "the ability to induce structural plasticity in many mecha- nisms (phototoxicity, injury, anesthesia/activity) lead to an interesting situation in which data supporting synaptic changes was more susceptible to artifact than was data supporting stability. Thus, the observation of no change in time-lapse imaging might still be easier to interpret than the evidence supporting dynamism." We agree with this statement and suggest it as a guideline for in vivo imaging and data interpretation. With the development of transgenic mouse models of various neurological diseases and the progress made in the field of in vivo TPM imaging, the time is ripe for answering long-standing questions with regards to structural changes in the process of learning and memory formation as well as in the recovery of function and rehabilitation following CNS injury.

Acknowledgments: This work was supported by the NIH grants to WBG and Ellison Foundation New Scholar Award in Aging to JG.

\section{REFERENCES}

1. Hubel DH, Wiesel TN, LeVay S. Plasticity of ocular dominance columns in monkey striate cortex. Philos Trans R Soc Lond B Biol Sci 1977;278:377-409.

2. Katz LC, Shatz CJ. Synaptic activity and the construction of cortical circuits. Science 1996;274:1133-1138.

3. Lichtman JW, Colman H. Synapse elimination and indelible memory. Neuron 2000;25:269-278.

4. Lund JS, Boothe RG, Lund RD. Development of neurons in the visual cortex (area 17) of the monkey (Macaca nemestrina): a Golgi study from fetal day 127 to postnatal maturity. J Comp Neurol 1977;176:149-188.

5. Rakic P, Bourgeois JP, Eckenhoff MF, Zecevic N, Goldman-Rakic PS. Concurrent overproduction of synapses in diverse regions of the primate cerebral cortex. Science 1986;232:232-235.

6. Bailey CH, Kandel ER. Structural changes accompanying memory storage. Annu Rev Physiol 1993;55:397-426.

7. Buonomano DV, Merzenich MM. Cortical plasticity: from synapses to maps. Annu Rev Neurosci 1998;21:149-186.

8. Darian-Smith C, Gilbert CD. Axonal sprouting accompanies functional reorganization in adult cat striate cortex. Nature 1994;368: 737-740.

9. Florence SL, Taub HB, Kaas JH. Large-scale sprouting of cortical connections after peripheral injury in adult macaque monkeys. Science 1998;282:1117-1121.

10. Jones EG, Pons TP. Thalamic and brainstem contributions to largescale plasticity of primate somatosensory cortex. Science 1998; 282:1121-1125.

11. Jones TA, Klintsova AY, Kilman VL, Sirevaag AM, Greenough WT. Induction of multiple synapses by experience in the visual cortex of adult rats. Neurobiol Learn Mem 1997;68:13-20.

12. Knott GW, Quairiaux C, Genoud C, Welker E. Formation of dendritic spines with GABAergic synapses induced by whisker stimulation in adult mice. Neuron 2002;34:265-273.

13. Ivanco TL, Greenough WT. Physiological consequences of morphologically detectable synaptic plasticity: potential uses for examining recovery following damage. Neuropharmacology 2000; 39:765-776.

14. Lichtman JW, Magrassi L, Purves D. Visualization of neuromuscular junctions over periods of several months in living mice. J Neurosci 1987;7:1215-1222.

15. Purves D, Hadley RD, Voyvodic JT. Dynamic changes in the dendritic geometry of individual neurons visualized over periods of up to three months in the superior cervical ganglion of living mice. J Neurosci 1986;6:1051-1060. 
16. Purves D, Voyvodic JT, Magrassi L, Yawo H. Nerve terminal remodeling visualized in living mice by repeated examination of the same neuron. Science 1987;238:1122-1126.

17. Feng G, Mellor RH, Bernstein M, et al. Imaging neuronal subsets in transgenic mice expressing multiple spectral variants of GFP. Neuron 2000;28:41-51.

18. Niell CM, Meyer MP, Smith SJ. In vivo imaging of synapse formation on a growing dendritic arbor. Nat Neurosci 2004;7:254260.

19. Wu GY, Zou DJ, Koothan T, Cline HT. Infection of frog neurons with vaccinia virus permits in vivo expression of foreign proteins. Neuron 1995;14:681-684.

20. Gan WB, Kwon E, Feng G, Sanes JR, Lichtman JW. Synaptic dynamism measured over minutes to months: age-dependent decline in an autonomic ganglion. Nat Neurosci 2003;6:956-960.

21. Grutzendler J, Kasthuri N, Gan WB. Long-term dendritic spine stability in the adult cortex. Nature 2002;420:812-816.

22. Trachtenberg JT, Chen BE, Knott GW, et al. Long-term in vivo imaging of experience-dependent synaptic plasticity in adult cortex. Nature 2002;420:788-794.

23. Sin WC, Haas K, Ruthazer ES, Cline HT. Dendrite growth increased by visual activity requires NMDA receptor and Rho GTPases. Nature 2002;419:475-480.

24. Denk W, Strickler JH, Webb WW. Two-photon laser scanning fluorescence microscopy. Science 1990;248:73-76.

25. Piston DW. Imaging living cells and tissues by two-photon excitation microscopy. Trends Cell Biol 1999;9:66-69.

26. Theer P, Hasan MT, Denk W. Two-photon imaging to a depth of 1000 micron in living brains by use of a Ti:Al2O3 regenerative amplifier. Opt Lett 2003;28:1022-1024.

27. Hua JY, Smear MC, Baier H, Smith SJ. Regulation of axon growth in vivo by activity-based competition. Nature 2005;434: 1022-1026.

28. Hua JY, Smith SJ. Neural activity and the dynamics of central nervous system development. Nat Neurosci 2004;7:327-332.

29. Meyer MP, Smith SJ. Evidence from in vivo imaging that synaptogenesis guides the growth and branching of axonal arbors by two distinct mechanisms. J Neurosci 2006;26:3604-3614.

30. Yuste R, Bonhoeffer T. Genesis of dendritic spines: insights from ultrastructural and imaging studies. Nat Rev Neurosci 2004;5: 24-34.

31. Meyer MP, Neill CM, Smith SJ. Brain imaging: how stable are synaptic connections? Curr Biol 2003;13:R180-R182.

32. Ottersen OP, Helm PJ. How hardwired is the brain? Nature 2002; 420:751-752.

33. Holtmaat AJ, Trachtenberg JT, Wilbrecht L, et al. Transient and persistent dendritic spines in the neocortex in vivo. Neuron 2005; 45:279-291.

34. Zuo Y, Lin A, Chang P, Gan WB. Development of long-term dendritic spine stability in diverse regions of cerebral cortex. Neuron $2005 \mathrm{a} ; 46: 181-189$.

35. Majewska AK, Newton JR, Sur M. Remodeling of synaptic structure in sensory cortical areas in vivo. J Neurosci 2006;26:30213029.

36. Mizrahi A, Crowley JC, Shtoyerman E, Katz LC. High-resolution in vivo imaging of hippocampal dendrites and spines. J Neurosci 2004;24:3147-3151.

37. Mizrahi A, Katz LC. Dendritic stability in the adult olfactory bulb. Nat Neurosci 2003;6:1201-1207.

38. Lee WC, Huang H, Feng G, et al. Dynamic remodeling of dendritic arbors in GABAergic interneurons of adult visual cortex. PLoS Biol 2006; 4:e29.

39. Yuste R, Bonhoeffer T. Morphological changes in dendritic spines associated with long-term synaptic plasticity. Annu Rev Neurosci 2001;24:1071-1089.

40. Zuo Y, Yang G, Kwon E, Gan WB. Long-term sensory deprivation prevents dendritic spine loss in primary somatosensory cortex. Nature 2005b;436:261-265.

41. Masliah E, Sisk A, Mallory M, Mucke L, Schenk D, Games D. Comparison of neurodegenerative pathology in transgenic mice overexpressing V717F $\beta$-amyloid precursor protein and Alzheimer's disease. J Neurosci 1996;16:5795-5811.
42. Kurt MA, Davies DC, Kidd M, et al. Neurodegenerative changes associated with $\beta$-amyloid deposition in the brains of mice carrying mutant amyloid precursor protein and mutant presenilin-1 transgenes. Exp Neurol 2001;171:59-71.

43. Brendza RP, Bacskai BJ, Cirrito JR, et al. Anti-A $\beta$ antibody treatment promotes the rapid recovery of amyloid-associated neuritic dystrophy in PDAPP transgenic mice. J Clin Invest 2005;115:428 433.

44. Scheibel AB, Tomiyasu U. Dendritic sprouting in Alzheimer's presenile dementia. Exp Neurol 1978;60:1-8.

45. Probst A, Basler V, Bron B, Ulrich J. Neuritic plaques in senile dementia of Alzheimer type: a Golgi analysis in the hippocampal region. Brain Res 1983;268:249-254.

46. Terry RD, Masliah E, Salmon DP, et al. Physical basis of cognitive alterations in Alzheimer's disease: synapse loss is the major correlate of cognitive impairment. Ann Neurol 1991;30:572-580.

47. Phinney AL, Deller T, Stalder M, et al. Cerebral amyloid induces aberrant axonal sprouting and ectopic terminal formation in amyloid precursor protein transgenic mice. J Neurosci 1999;19:85528559.

48. Knowles RB, Gomez-Isla T, Hyman BT. A $\beta$ associated neuropil changes: correlation with neuronal loss and dementia. J Neuropathol Exp Neurol 1998;57:1122-1130.

49. Le R, Cruz L, Urbanc B, et al. Plaque-induced abnormalities in neurite geometry in transgenic models of Alzheimer's disease: implications for neural system disruption. J Neuropathol Exp Neurol 2001;60:753-758.

50. Urbanc B, Cruz L, Le R, et al. Neurotoxic effects of thioflavin S-positive amyloid deposits in transgenic mice and Alzheimer's disease. Proc Natl Acad Sci U S A 2002;99:13990-13995.

51. Cummings BJ, Pike CJ, Shankle R, Cotman CW. $\beta$-amyloid deposition and other measures of neuropathology predict cognitive status in Alzheimer's disease. Neurobiol Aging 1996;17:921-933.

52. Knowles RB, Wyart C, Buldyrev SV, et al. Plaque-induced neurite abnormalities: implications for disruption of neural networks in Alzheimer's disease. Proc Natl Acad Sci U S A 1999;96:52745279.

53. Gomez-Isla T, Hollister R, West H, et al. Neuronal loss correlates with but exceeds neurofibrillary tangles in Alzheimer's disease. Ann Neurol 1997;41:17-24.

54. Crystal H, Dickson D, Fuld P, et al. Clinico-pathologic studies in dementia: nondemented subjects with pathologically confirmed Alzheimer's disease. Neurology 1988;38:1682-1687.

55. Arriagada PV, Marzloff K, Hyman BT. Distribution of Alzheimertype pathologic changes in nondemented elderly individuals matches the pattern in Alzheimer's disease [see comments]. Neurology 1992;42:1681-1688.

56. Hsia AY, Masliah E, McConlogue L, et al. Plaque-independent disruption of neural circuits in Alzheimer's disease mouse models. Proc Natl Acad Sci U S A 1999;96:3228-3233.

57. Lacor PN, Buniel MC, Chang L, et al. Synaptic targeting by Alzheimer's-related amyloid $\beta$ oligomers. J Neurosci 2004;24: 10191-10200.

58. Tsai J, Grutzendler J, Duff K, Gan WB. Fibrillar amyloid deposition leads to local synaptic abnormalities and breakage of neuronal branches. Nat Neurosci 2004;7:1181-1183.

59. Spires TL, Meyer-Luehmann M, Stern EA, et al. Dendritic spine abnormalities in amyloid precursor protein transgenic mice demonstrated by gene transfer and intravital multiphoton microscopy. J Neurosci 2005;25:7278-7287.

60. McGeer PL, McGeer EG. Inflammation and the degenerative diseases of aging. Ann N Y Acad Sci 2004;1035:104-116.

61. Jung S, Aliberti J, Graemmel P, et al. Analysis of fractalkine receptor CX(3)CR1 function by targeted deletion and green fluorescent protein reporter gene insertion. Mol Cell Biol 2000;20: $4106-4114$.

62. Zuo Y, Lubischer JL, Kang H, et al. Fluorescent proteins expressed in mouse transgenic lines mark subsets of glia, neurons, macrophages, and dendritic cells for vital examination. J Neurosci 2004; 24:10999-11009.

63. Davalos D, Grutzendler J, Yang G, et al. ATP mediates rapid microglial response to local brain injury in vivo. Nat Neurosci 2005;8:752-758. 
64. Zhang S, Boyd J, Delaney K, Murphy TH. Rapid reversible changes in dendritic spine structure in vivo gated by the degree of ischemia. J Neurosci 2005;25:5333-5338.

65. Zhang ZG, Zhang L, Ding G, et al. A model of mini-embolic stroke offers measurements of the neurovascular unit response in the living mouse. Stroke 2005;36:2701-2704.

66. Schaffer CB, Friedman B, Nishimura N, et al. Two-photon imaging of cortical surface microvessels reveals a robust redistribution in blood flow after vascular occlusion. PLoS Biol 2006;4:E22.

67. Jin K, Minami M, Lan JQ, et al. Neurogenesis in dentate subgranular zone and rostral subventricular zone after focal cerebral ischemia in the rat. Proc Natl Acad Sci U S A 2001;98:4710-4715.

68. Zhang R, Zhang Z, Zhang C, et al. Stroke transiently increases subventricular zone cell division from asymmetric to symmetric and increases neuronal differentiation in the adult rat. J Neurosci 2004;24:5810-5815.

69. Huttenlocher PR. Morphometric study of human cerebral cortex development. Neuropsychologia 1990;28:517-527.
70. Markus EJ, Petit TL. Neocortical synaptogenesis, aging, and behavior: lifespan development in the motor-sensory system of the rat. Exp Neurol 1987;96:262-278.

71. Matsuzaki M, Ellis-Davies GC, Nemoto T, Miyashita Y, Iino M, Kasai H. Dendritic spine geometry is critical for AMPA receptor expression in hippocampal CA1 pyramidal neurons. Nat Neurosci 2001;4:1086-1092.

72. Dailey ME, Smith SJ. The dynamics of dendritic structure in developing hippocampal slices. J Neurosci 1996;16:29832994.

73. Fiala JC, Feinberg M, Popov V, Harris KM. Synaptogenesis via dendritic filopodia in developing hippocampal area CA1. J Neurosci 1998;18:8900-8911.

74. Ziv NE, Smith SJ. Evidence for a role of dendritic filopodia in synaptogenesis and spine formation. Neuron 1996;17:91-102.

75. Kasthuri N, Lichtman JW. Structural dynamics of synapses in living animals. Curr Opin Neurobiol 2004;14:105-111. 